\title{
Peroxisome Proliferation
}

National Cancer Institute

\section{Source}

National Cancer Institute. Peroxisome Proliferation. NCI Thesaurus. Code C18933.

Associated with hepatocellular carcinomas, Peroxisome Proliferation occurs in epigenetic "non-genotoxic" responses of hepatocytes to diverse peroxisome proliferator chemicals and involves, probably by a steroid superfamily receptor-based mechanism (PPARs),

altered expression of cellular growth and/or differentiation genes, induction of peroxisomal enzymes, and possibly oxidative stress. 